\section{International Scientific Journal Theoretical \& Applied Science}

\author{
p-ISSN: 2308-4944 (print) e-ISSN: 2409-0085 (online) \\ Year: 2017 Issue: 02 Volume: 46
}

Published: $28.02 .2017 \quad \underline{\text { http://T-Science.org }}$

SECTION 6. Metallurgy and energy.
E.S. Belyaev

Candidate of technical Sciences, associate professor of «Materials science, technology of materials and heat treatment of metals» Nizhny Novgorod State Technical University n.a. R.E. Alekseev

S.S. Durdyeva Master student of the Department «Materials science, technology of materials and heat treatment of metals»» Nizhny Novgorod State Technical University n.a. R.E. Alekseev

N. V. Makarov

Postgraduate student of the Department «Materials science, technology of materials and heat treatment of metals»)

Nizhny Novgorod State Technical University n.a. R.E. Alekseev

\title{
LASER TREATMENT EFFECTS ON GEOMETRIC DIMENSIONS OF MELTING AND HEAT AFFECTED ZONES AT PROCESSING OF SINTERED POROUS IRON
}

\footnotetext{
Abstract: The article considers the laser radiation effects on the formation of the melting zone and the heataffected zone of the porous sintered iron with using the method of mathematical planning experiments. You can see the results of influence of factors, their double and triple interaction on the geometric characteristics of the melting and the heat-affected zones. It is analyzed the role of the energy flux density (interaction of laser power density and displacement speed of the laser beam on the surface), the porosity and their triple interaction - structurally energy parameter at processing porous iron.

Key words: the melting zone, the heat-affected zone, laser treatment, the processing mode, the power density, the speed of movement of the beam, the energy flux density, the mathematical planning experiment

Language: Russian

Citation: Belyaev ES, Durdyeva SS, Makarov NV (2017) LASER TREATMENT EFFECTS ON GEOMETRIC DIMENSIONS OF MELTING AND HEAT AFFECTED ZONES AT PROCESSING OF SINTERED POROUS IRON. ISJ Theoretical \& Applied Science, 02 (46): 143-154.

Soi: http://s-o-i.org/1.1/TAS-02-46-26 Doi: croskef https://dx.doi.org/10.15863/TAS.2017.02.46.26

\section{ВЛИЯНИЕ ЛАЗЕРНОЙ ОБРАБОТКИ НА ГЕОМЕТРИЧЕСКИЕ РАЗМЕРЫ ЗОН ПЛАВЛЕНИЯ И ТЕРМИЧЕСКОГО ВЛИЯНИЯ ПРИ ОБРАБОТКЕ СПЕЧЕННОГО ПОРИСТОГО ЖЕЛЕЗА}

Аннотация: В работе рассмотрено воздействие лазерного излучения на формирование зонь плавления и зоны термического влияния пористого спеченного железа с применением метода математического планирования экспериментов. Показаны влияния факторов, их двойных и тройных взаимодействий на геометрические характеристики зон плавления и термического влияния. Рассмотрена роль плотности потока энергии (взаимодействие плотности мощңности лазерного излучения и скорости перемещения лазерного луча по поверхности), пористости и их тройного взаимодействия - структурноэнергетического параметра обработки пористого железа.

Ключевые слова: зона плавления, зона термического влияния, лазерная обработка, режим обработки, плотность мощности, скорость перемещения луча, плотность потока энергии, математическое планирование эксперимента
} 


\section{Введение}

Пористые материалы имеют равномерную объемнораспределенную пористость, которая является важнейшей эксплуатационной характеристикой и структурной составляющей таких материалов. Наличие пористости определяет направление применения таких материалов в технике, приборостроении и т.д.[1].

Объем пор в пористом материале определяет его назначение: $10 . .13 \%$ - фрикционные материалы; $15 \ldots 35 \%$ - антифрикционные материалы; 20...50\% - фильтры; 50...98\% высокопористые или пеноматериалы [1]. Увеличение объемной доли пор в материале приводит к изменению теплофизических [1-3] (теплопроводность, температуропроводность), оптических [4] (коэффициенты отражения и поглощения), электрических [2, 3] (удельная проводимость, удельное электросопротивление) и механических [1-3] свойств материалов. Пористость снижает все механические свойства по сравнению с аналогичным по химическому составу беспористым материалом [1-3].

Воздействие на поверхность пористого тела лазерного излучения инициирует развитие усадочных процессов приводящих к снижению пористости [5-9]. Динамика перехода от открытой к закрытой пористости, уменьшения размеров пор и их количества зависят от энергетического режима лазерной обработки [69]. Очевидно, что уменьшение пористости на поверхности приведет к росту ее физикомеханических свойств. Однако локальное и значительное снижение пористости в пористом теле вызывает значительные напряжения, обусловленные протекающими усадочными процессами. В [6-9] установлено наличие «зоны пониженной прочности» в зоне перехода от литой сварочной ванны к пористому материалу после воздействия лазерного излучения.

В свете вышеизложенного изучение воздействия лазерного излучения на поверхность пористых материалов является актуальной научно-практической задачей, решение которой позволит с помощью лазерной обработки формировать структуру и управлять свойствами пористых материалов.
Целью данного исследования является изучение воздействия лазерного излучения на поверхность спеченных пористых образцов на основе порошка железа, полученных методами порошковой металлургии.

\section{Материалы и оборудование}

Образцы для лазерной обработки поверхности изготовлены методом порошковой металлургии (прессование + спекание). Прессовки изготовлены из порошка восстановленного карбонильного железа марки ВК-1 (ТУ 2436- 005-74439740-14). Гранулометрический и химический состав порошка представлен в табл. 1, морфология частиц порошка показана на рисунке 1.

Прессование проводили в закрытой прессформе с проставкой, ограничивающей ход пуансона, обеспечивающей получение неспеченных прессовок диаметром 15 мм и высотой 15 мм. Изменением массы навески производилось регулирование пористости неспеченных прессовок. Спекание производили в атмосфере осушенного водорода при температуре $1100^{\circ} \mathrm{C}$ в течении 120 минут. После спекания получены три вида образцов с различной пористостью соответственно 22, 30 и $38 \%$.

Выбор порошка железа марки ВК-1 для проведения исследований обусловлен его высокой дисперсностью, низким содержанием углерода и других примесей, что позволит рассмотреть влияние лазерной обработки на пористое тело в отсутствии мартенситного превращения.

Обработку поверхности выполняли с помощью установки «Латус-31» представляющей собой молекулярный газовый $\mathrm{CO}_{2}$ лазер (длина волны излучения $\rightleftharpoons 10,6$ мкм) на основе компактного конвективного оптического квантового генератора «Карат». На поверхность обрабатываемых образцов наносилось поглощающее покрытие - художественная гуашь с желтым железноокисным пигментом Ж-1 ГОСТ 18172-80.

Просмотр микроструктуры и измерения параметров отклика выполнены на цифровом микроскопе Keyence VHX-1000.

\section{Гранулометрический и химический состав порошка ВК-1}

Таблица 1

\begin{tabular}{|c|c|c|c|c|c|c|c|}
\hline \multicolumn{2}{|c|}{ Гранулометрический состав, мкм, не } & \multicolumn{4}{|c|}{ Массовая доля химических элементов, не более } \\
\hline $\mathrm{X} 10$ & $\mathrm{X} 50$ & $\mathrm{X} 90$ & $F e, \%$ & $C, \%$ & $N, \%$ & $O, \%$ & $S, \%$ \\
\hline 4 & 8 & 18 & основа & $0,02-0,1$ & 0,02 & 0,3 & 0,005 \\
\hline
\end{tabular}




\begin{tabular}{|c|c|c|c|c|c|c|}
\hline Impact Factor: & $\begin{array}{l}\text { ISRA (India) } \\
\text { ISI (Dubai, UAF } \\
\text { GIF (Australia) } \\
\text { JIF }\end{array}$ & $\begin{array}{l}=1.344 \\
=0.829 \\
=0.564 \\
=1.500\end{array}$ & $\begin{array}{l}\text { SIS (USA) } \\
\text { PИНЦ (Russia) } \\
\text { ESJI (KZ) } \\
\text { SJIF (Morocco) }\end{array}$ & $\begin{array}{l}=0.912 \\
=0.234 \\
=1.042 \\
=\mathbf{2 . 0 3 1}\end{array}$ & $\begin{array}{l}\text { ICV (Poland) } \\
\text { PIF (India) } \\
\text { IBI (India) }\end{array}$ & $\begin{array}{l}=6.630 \\
=1.940 \\
=4.260\end{array}$ \\
\hline
\end{tabular}

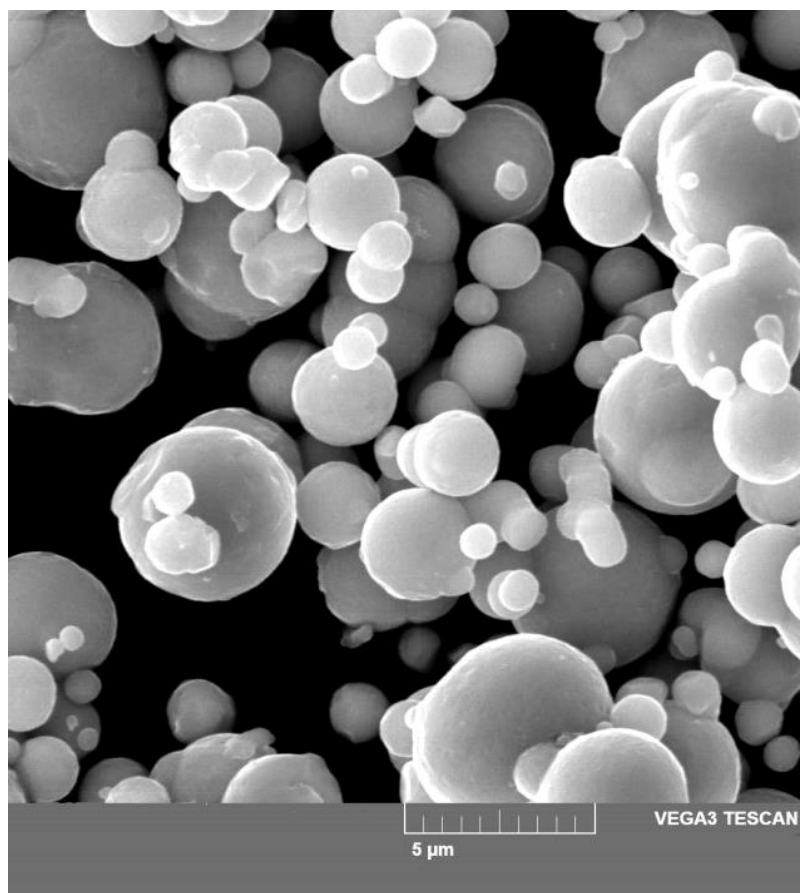

Рисунок 1 - Морфология частиц порошка карбонильного железа ВК-1.

\section{Планирование эксперимента и} разработка регрессионных моделей

Для разработки матрицы планирования экспериментов были выбраны три изменяемых фактора:

$x_{1} \quad$ - плотность мощности лазерного

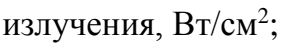

$x_{2}$ - скорость перемещения лазерного излучения, мм/с;

$x_{3}$ - пористость материала, \%.

Три фактора позволяют построить план $N=2^{K}$, где $K$ число факторов, 2 - число уровней (верхний «+1» и нижний «-1»). План $2^{3}$ содержит $N=2^{3}=8$ - опытов на верхнем и нижнем уровне и дополнительные три параллельных опыта на основном уровне. Параллельные опыты

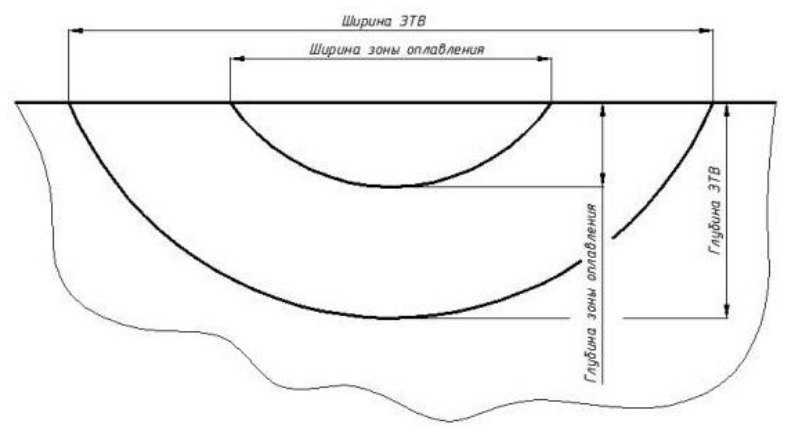

a) позволяют оценить точность построенной модели и ее линейность за счет введения в матрицу фиктивного фактора «Центр».

На основном уровне факторы имеют следующие натуральные значения: $x_{1}-1 \cdot 10^{4}$ $\mathrm{Bт} / \mathrm{cm}^{2}, x_{2}-15 \mathrm{~mm} / \mathrm{c}, x_{3}-30 \%$. Интервалы изменения факторов в натуральном масштабе: $\Delta x_{1}- \pm 0,2 \cdot 10^{4} \mathrm{BT} / \mathrm{cm}^{2}, \Delta x_{2}- \pm 5 \mathrm{~mm} / \mathrm{c}, \Delta x_{3}- \pm 8$ $\%$.

В качестве параметров отклика системы были приняты ширина, глубина зоны плавления и зоны термического влияния (ЗТВ). Схема измерений и микроструктура зоны термического влияния показана на рисунке 2 .

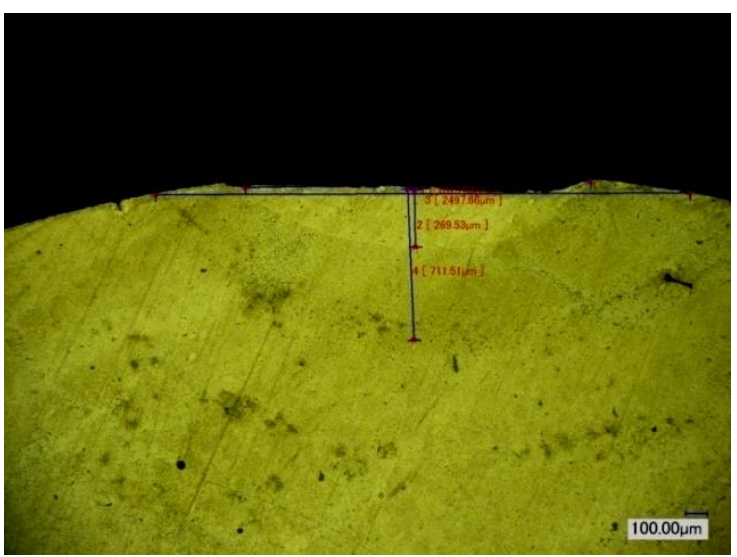

б)

Рисунок 2 - Схема измерений параметров отклика (а) и микроструктура зоны термического влияния $(\boldsymbol{0}, \Pi=22 \%, \mathbf{x 1 0 0})$ 


\begin{tabular}{|c|c|c|c|c|c|c|}
\hline Impact Factor: & $\begin{array}{l}\text { ISRA (India) } \\
\text { ISI (Dubai, UAF } \\
\text { GIF (Australia) } \\
\text { JIF }\end{array}$ & $\begin{array}{l}=1.344 \\
=0.829 \\
=0.564 \\
=1.500\end{array}$ & $\begin{array}{l}\text { SIS (USA) } \\
\text { PИНЦ (Russia) } \\
\text { ESJI (KZ) } \\
\text { SJIF (Morocco }\end{array}$ & $\begin{array}{l}=0.912 \\
=0.234 \\
=1.042 \\
=\mathbf{2 . 0 3 1}\end{array}$ & $\begin{array}{l}\text { ICV (Poland) } \\
\text { PIF (India) } \\
\text { IBI (India) }\end{array}$ & $\begin{array}{l}=6.630 \\
=1.940 \\
=4.260\end{array}$ \\
\hline
\end{tabular}

Матрица планирования и результаты измерений представлены в таблице 2.
Разработанные регрессионные уравнения в кодовом масштабе имеют вид:

$$
\begin{aligned}
& y_{1}=2459,2-1218,7 x_{L}+830,9 x_{1}-741,3 x_{2}+468,6 x_{3}-338 x_{1} x_{2}+427,6 x_{1} x_{2} x_{3} \\
& y_{2}=311,3-165,9 x_{L}+78 x_{1}-71,1 x_{2}+91,3 x_{3}+50,4 x_{1} x_{2} x_{3} \\
& y_{3}=3330,4-444,1 x_{L}+255,2 x_{1}-150,8 x_{2}+238,3 x_{3}-418,6 x_{1} x_{2}+57 x_{2} x_{3}-47,8 x_{1} x_{2} x_{3} \\
& y_{4}=679,4+149,2 x_{3}-139,9 x_{1} x_{2}
\end{aligned}
$$

\begin{tabular}{|c|c|c|c|c|c|c|c|c|c|c|c|}
\hline \multirow{3}{*}{$\begin{array}{c}\text { № } \\
\text { опыта } \\
\quad i\end{array}$} & \multicolumn{7}{|c|}{ Значения факторов } & \multicolumn{4}{|c|}{ Параметры отклика системы } \\
\hline & \multirow{2}{*}{$\begin{array}{l}\text { «Цен } \\
m p » » \\
\text { код. }\end{array}$} & \multicolumn{2}{|c|}{$x_{1}^{*}$} & \multicolumn{2}{|c|}{$x_{2}$} & \multicolumn{2}{|c|}{$x_{3}$} & \multirow{2}{*}{$\begin{array}{c}y_{1} \text { - ширина } \\
\text { зоны } \\
\text { оплавления, } \\
\text { мкм }\end{array}$} & \multirow{2}{*}{$\begin{array}{c}y_{2} \text { - глубина } \\
\text { зоны } \\
\text { оплавления, } \\
\text { мкм }\end{array}$} & \multirow{2}{*}{$\begin{array}{c}y_{3}- \\
\text { ширина } \\
\text { ЗТВ, } \\
\text { мкм }\end{array}$} & \multirow{2}{*}{$\begin{array}{c}y_{4}- \\
\text { глубина } \\
\text { ЗТВ, } \\
\text { мкм }\end{array}$} \\
\hline & & код. & нат., \% & код. & $\begin{array}{c}\text { нат. } \\
, \%\end{array}$ & код. & $\begin{array}{l}\text { нат } \\
., \%\end{array}$ & & & & \\
\hline \multicolumn{12}{|c|}{ Опыты на верхнем и нижнем уровне } \\
\hline 1 & 1 & 1 & $1,2 \cdot 10^{4}$ & 1 & 20 & 1 & 38 & 1971,75 & 297,09 & 2818,85 & 647,72 \\
\hline 2 & 1 & -1 & $0,8 \cdot 10^{4}$ & 1 & 20 & 1 & 38 & 0,00000 & 0,0000 & 3242,76 & 1037,69 \\
\hline 3 & 1 & 1 & $1,2 \cdot 10^{4}$ & -1 & 10 & 1 & 38 & 3226,57 & 379,94 & 3939,16 & 908,26 \\
\hline 4 & 1 & -1 & $0,8 \cdot 10^{4}$ & -1 & 10 & 1 & 38 & 1613,03 & 269,53 & 2497,66 & 711,51 \\
\hline 5 & 1 & 1 & $1,2 \cdot 10^{4}$ & 1 & 20 & -1 & 22 & 0,00000 & 0,0000 & 2325,45 & 368,15 \\
\hline 6 & 1 & -1 & $0,8 \cdot 10^{4}$ & 1 & 20 & -1 & 22 & 0,00000 & 0,0000 & 2554,97 & 651,64 \\
\hline 7 & 1 & 1 & $1,2 \cdot 10^{4}$ & -1 & 10 & -1 & 22 & 3062,26 & 216,54 & 3482,78 & 670,57 \\
\hline 8 & 1 & -1 & $0,8 \cdot 10^{4}$ & -1 & 10 & -1 & 22 & 0,00000 & 0,0000 & 2228,89 & 421,21 \\
\hline \multicolumn{12}{|c|}{ Параллельные опыты на основном уровне } \\
\hline 9 & 0 & 0 & $1 \cdot 10^{4}$ & 0 & 15 & 1 & 30 & 2325,79 & 265,29 & 3300,18 & 645,52 \\
\hline 10 & 0 & 0 & $1 \cdot 10^{4}$ & 0 & 15 & 1 & 30 & 2559,34 & 367,17 & 3361,36 & 810,76 \\
\hline 11 & 0 & 0 & $1 \cdot 10^{4}$ & 0 & 15 & 1 & 30 & 2473,57 & 301,36 & 3329,57 & 600,65 \\
\hline
\end{tabular}

\section{Матрица планирования экспериментов и результаты измерений}

Таблица 2

В полученные уравнения 1-4 вошли коэффициенты уровень значимости $p$ менее 0,05 . Уровень значимости $p$ вычислен на основании критерия Фишера. Скорректированный коэффициент детерминации $R^{2}$ полученных уравнений 1-4 не ниже 0,88. После выполнения соответствующих расчетов выяснилось, что гипотеза о статистической значимости фиктивного фактора «Центр» не отвергается в регрессионных уравнениях по параметрам оптимизации $y_{1}, y_{2}$ и $y_{3}$. Статистическая значимость фиктивного фактора «Центр» свидетельствует о нелинейном характере регрессионных уравнений 1-3. В данном случае фактор «Центр» линейно возрастает от значения «0»к «1» при движении от центра плана к его краям.
Наглядно демонстрируют влияния различных факторов и их взаимодействий на параметры оптимизации $y_{1}, \quad-y_{4}$ карты стандартизированных эффектов Парето, представленные на рисунках 3-6.

\section{Обсуждение полученных результатов}

Строение зоны лазерного воздействия в выполненных опытах №№1-8 различно, в опытах №o2, 5, 6, 8 отсутствует зона плавления, а ЗТВ присутствует. В остальных опытах имеется и зона плавления и ЗТВ.

На ширину зоны плавления сильно влияет плотность мощности лазерного излучения и скорость обработки, т.е. фактически время воздействия лазерного излучения на поверхность материала:

ISPC Technology and science, 


\begin{tabular}{|c|c|c|c|c|c|c|}
\hline Impact Factor: & $\begin{array}{l}\text { ISRA (India) } \\
\text { ISI (Dubai, UAE } \\
\text { GIF (Australia) } \\
\text { JIF }\end{array}$ & $\begin{array}{l}=1.344 \\
=0.829 \\
=0.564 \\
=1.500\end{array}$ & $\begin{array}{l}\text { SIS (USA) } \\
\text { PИНЦ (Russia) } \\
\text { ESJI (KZ) } \\
\text { SJIF (Morocco) }\end{array}$ & $\begin{array}{l}=0.912 \\
=0.234 \\
=1.042 \\
=2.031\end{array}$ & $\begin{array}{l}\text { ICV (Poland) } \\
\text { PIF (India) } \\
\text { IBI (India) }\end{array}$ & $\begin{array}{l}=6.630 \\
=1.940 \\
=4.260\end{array}$ \\
\hline
\end{tabular}

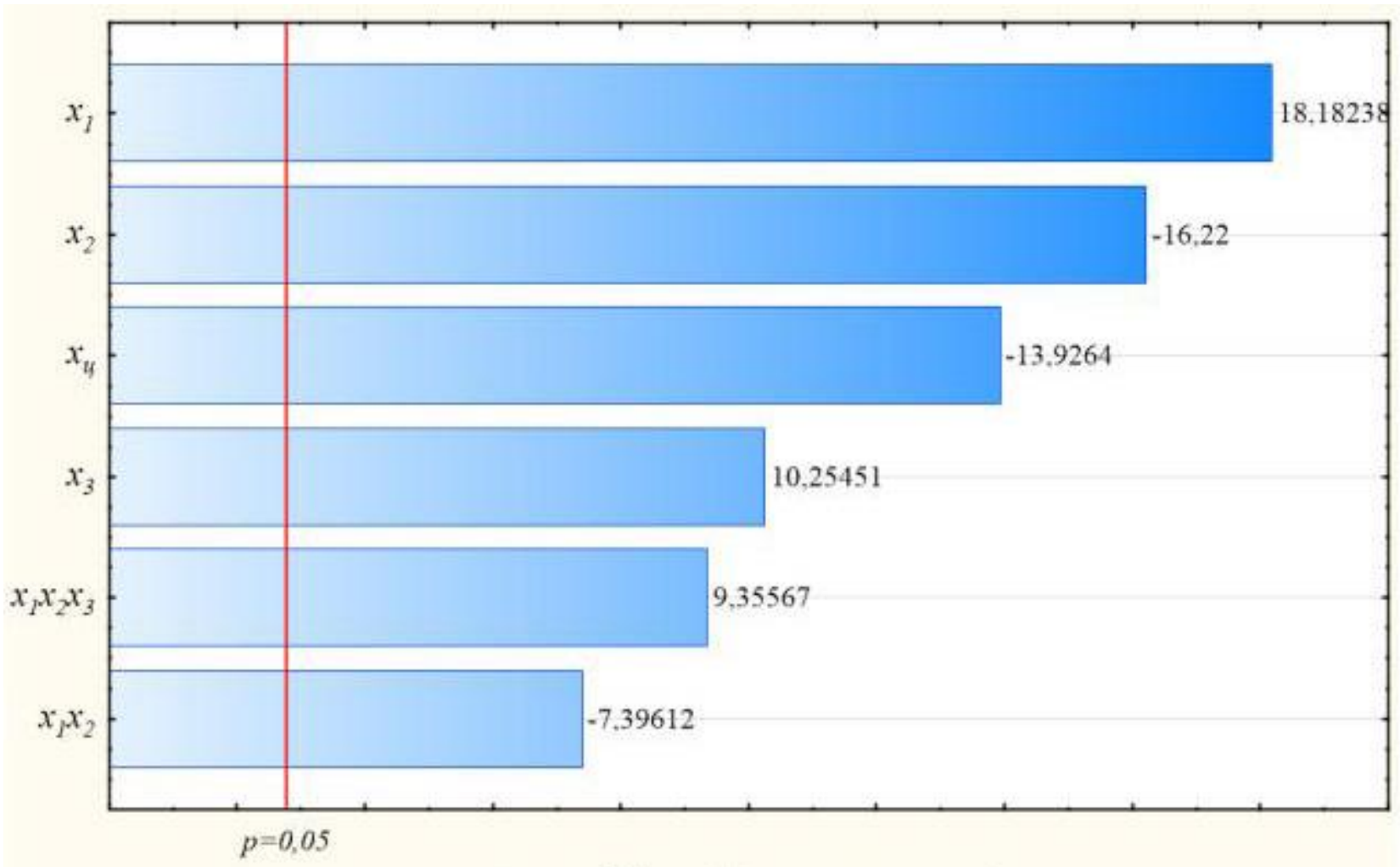

Оценка эффекта (абссолютное значение)

Рисунок 3 - Карта стандартизированных эффектов Парето по параметру оптимизации «Ширина зоны

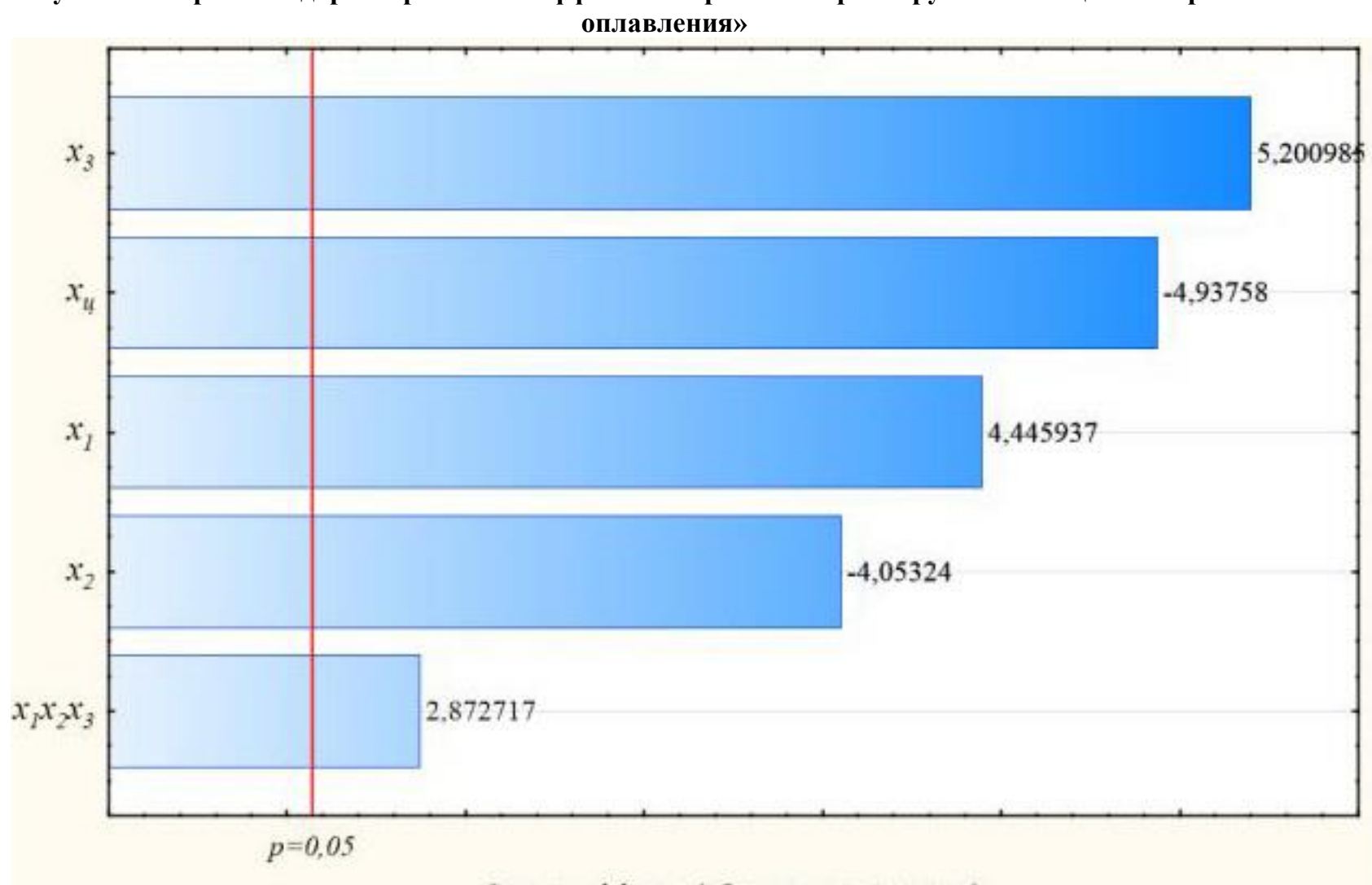

Оценка эффекта (абосолютное значение)

Рисунок 4 - Карта стандартизированных эффектов Парето по параметру оптимизации «Глубина зоны оплавления» 


\begin{tabular}{|c|c|c|c|c|c|c|}
\hline Impact Factor: & $\begin{array}{l}\text { ISRA (India) } \\
\text { ISI (Dubai, UAE } \\
\text { GIF (Australia) } \\
\text { JIF }\end{array}$ & $\begin{array}{l}=1.344 \\
=0.829 \\
=0.564 \\
=1.500\end{array}$ & $\begin{array}{l}\text { SIS (USA) } \\
\text { PИНЦ (Russia) } \\
\text { ESJI (KZ) } \\
\text { SJIF (Morocco) }\end{array}$ & $\begin{array}{l}=0.912 \\
=0.234 \\
=1.042 \\
=2.031\end{array}$ & $\begin{array}{l}\text { ICV (Poland) } \\
\text { PIF (India) } \\
\text { IBI (India) }\end{array}$ & $\begin{array}{l}=6.630 \\
=1.940 \\
=4.260\end{array}$ \\
\hline
\end{tabular}

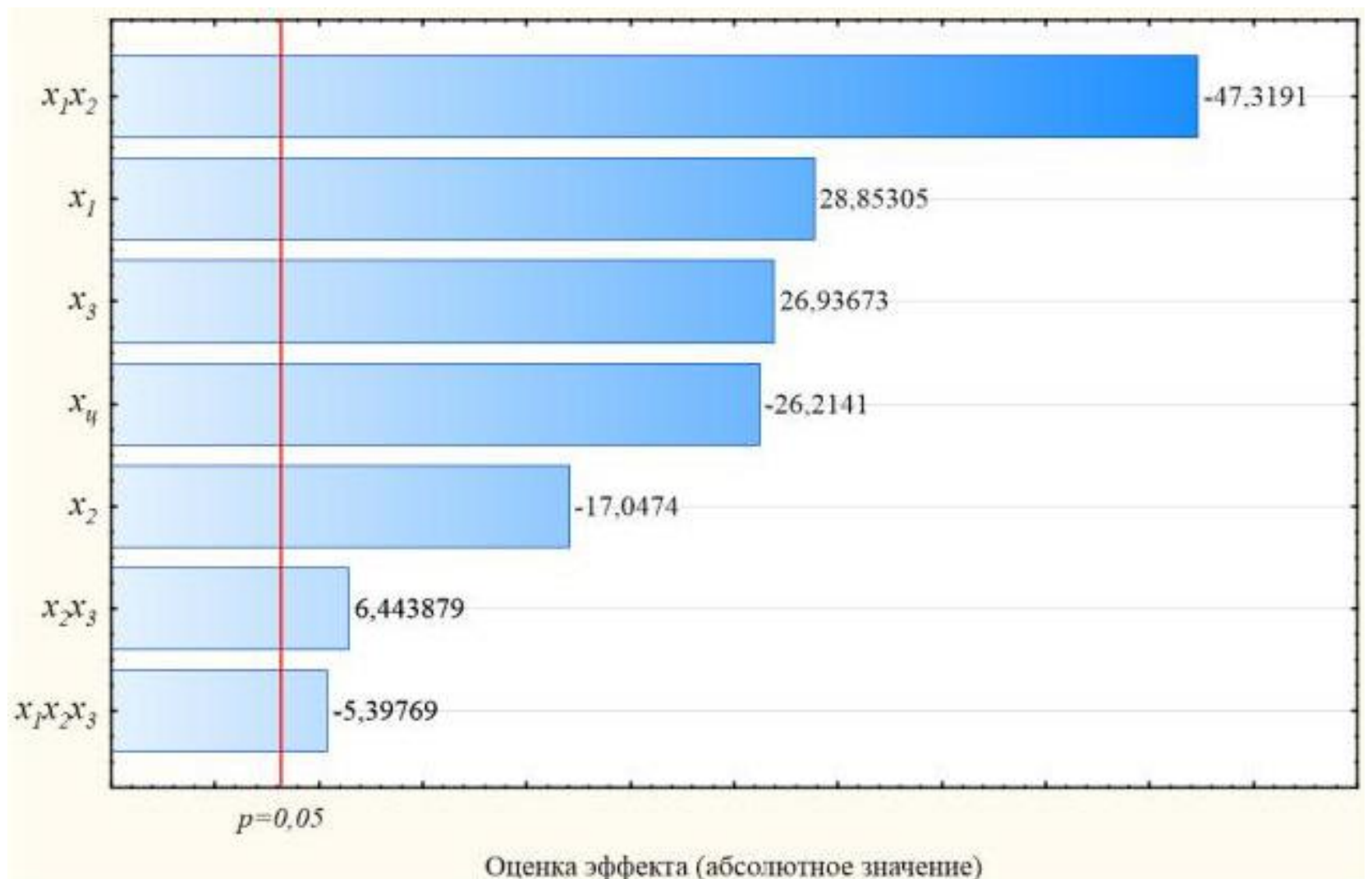

Рисунок 5 - Карта стандартизированных эффектов Парето по параметру оптимизации «Ширина 3TB»

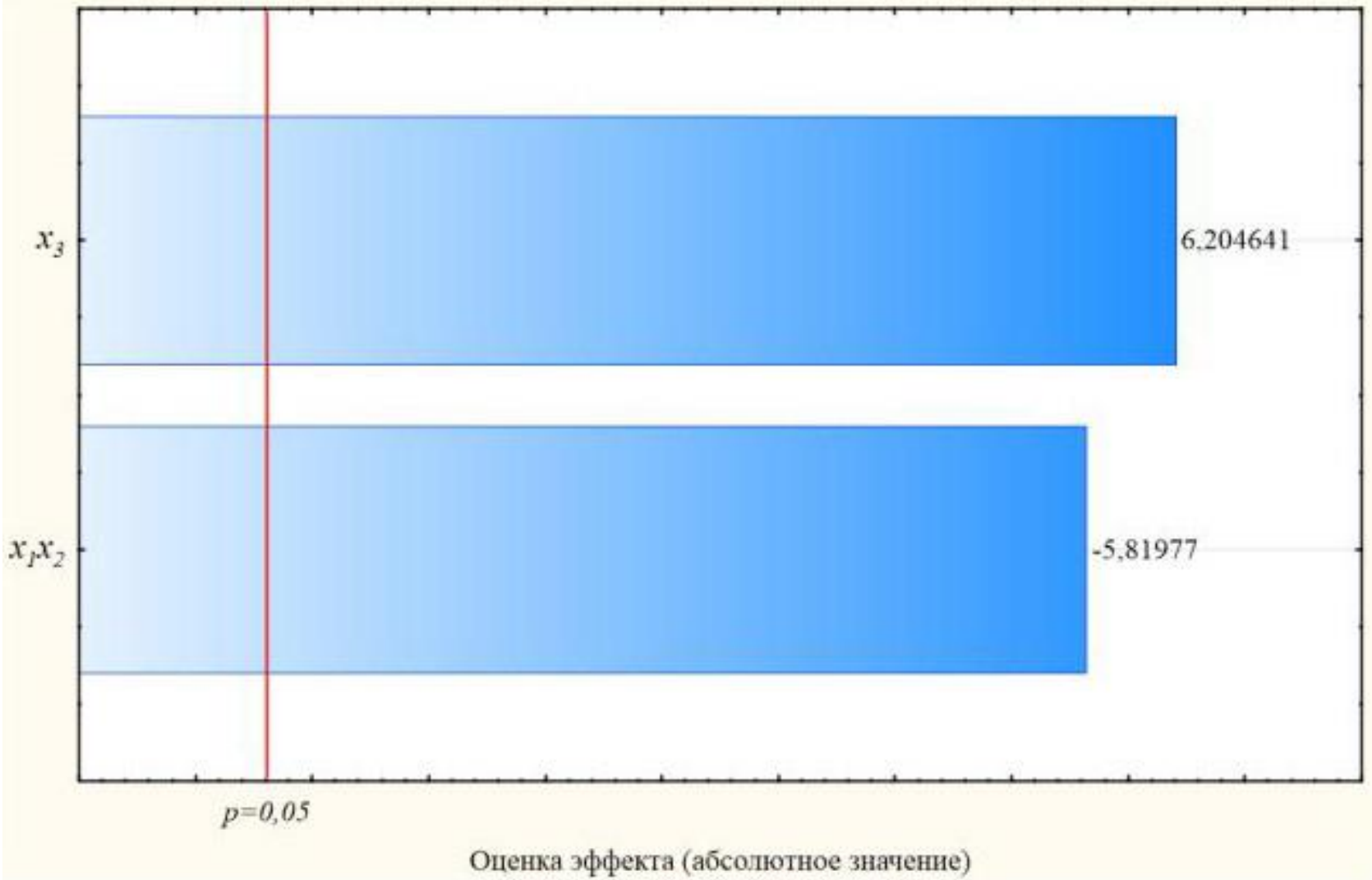

Рисунок 6 - Карта стандартизированных эффектов Парето по параметру оптимизации «Глубина 3TB» 
где $\tau$ - время обработки, с;

$$
\tau=d / v
$$

$d$ - диаметр «лазерного пятна» на обрабатываемой поверхности, мм;

$v$ - скорость перемещения лазерного пятна по поверхности, мм/с.

С ростом плотности мощности и времени воздействия лазерного излучения на поверхность (с уменьшением скорости обработки) ширина зоны плавления увеличивается (уравнение 1), что
(5)

хорошо согласуется с результатами [10] для беспористых материалов.

Коэффициент взаимодействия плотности мощности и скорости обработки имеет отрицательный знак и соответственно уменьшает ширину зоны плавления (уравнение 1).

Размерность парного взаимодействия плотности мощности и скорости обработки через время воздействия лазерного излучения на поверхность можно представить как:

$$
x_{1} \cdot x_{2}=\left[\frac{B m}{c M^{2}}\right] \cdot\left[\frac{c}{1}\right]=\left[\frac{Д ж \cdot c}{c \cdot c M^{2}}\right]=\left[\frac{Д ж}{c M^{2}}\right]
$$

Таким образом, с ростом времени воздействия лазерного излучения на поверхность (т.е. скорость обработки становится ниже) плотность потока энергии увеличивается, что ведет к снижению глубины оплавленного слоя.
Такое явление можно объяснить развитием на поверхности плазменных процессов [10, 11]. На рисунке 7 представлены изолинии ширины оплавленной зоны в координатах плотность мощности - скорость обработки.

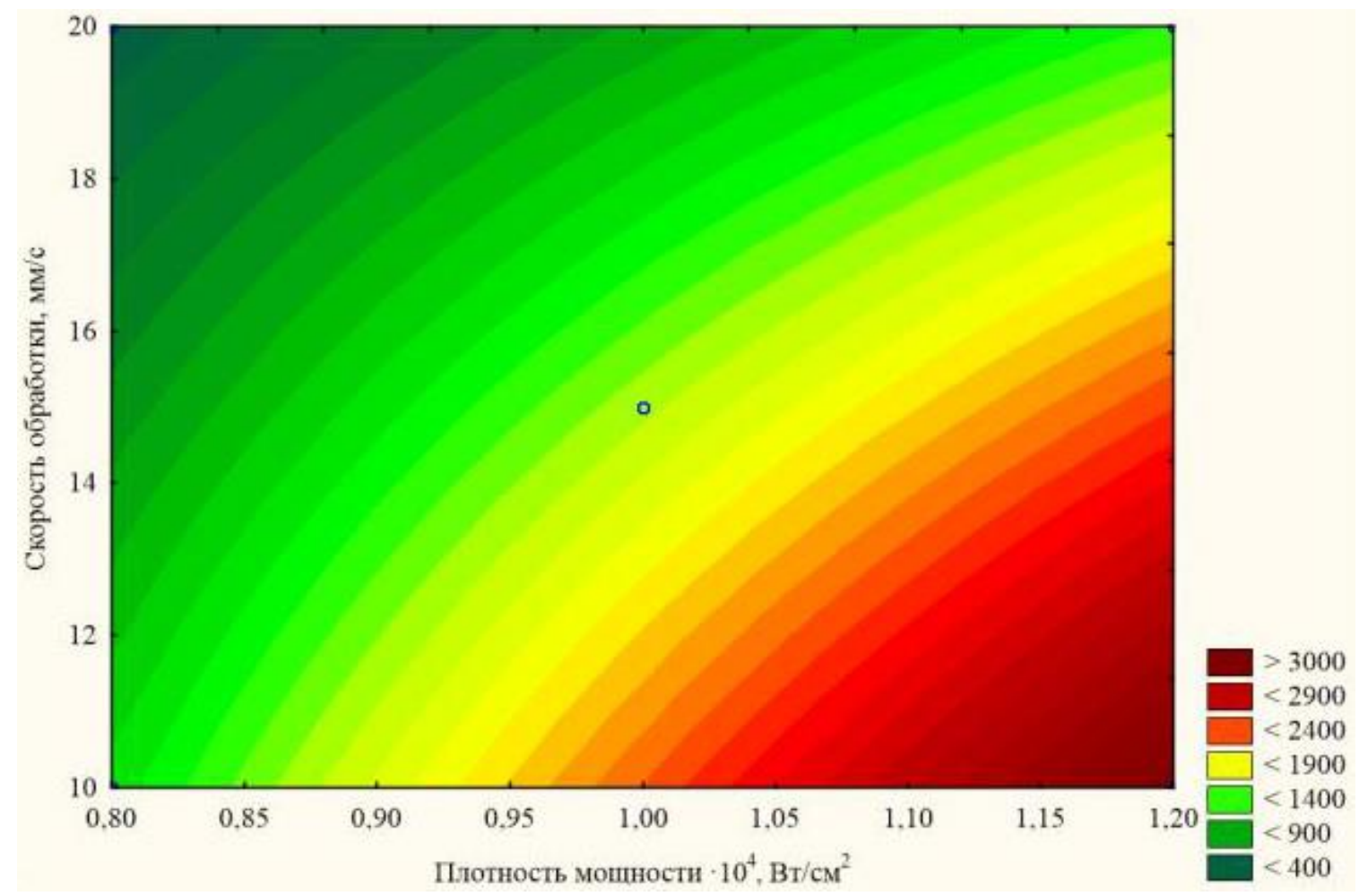

Рисунок 7 - Проекции изолиний ширины оплавленной зоны на плоскость $\left(x_{u}=0,73, \Pi=30 \%\right)$

Коэффициент, отражающий влияние пористости прессовок на процесс формирования оплавленной зоны при лазерном облучении поверхности, имеет положительный знак (уравнение 1), т.е. с ростом пористости ширина зоны плавления увеличивается, что согласуется с данными [5].
Пористость теплопроводность теплопроводность существенно материала. пористого снижает Оценить материала, возможно используя следующее соотношение [2, 3]:

$$
\lambda_{\Pi}=\lambda_{K}(1-1,5 \Pi)
$$


где $\square_{\Pi}$ - теплопроводность пористого материала, $\mathrm{BT} / \mathrm{M} \cdot \mathrm{K}$

$\square_{\kappa} \quad$ - теплопроводность компактного материала аналогичного химического состава, $\mathrm{BT} / \mathrm{M} \cdot \mathrm{K}$; доля).

П - пористость в долях единицы (объемная

$$
\square_{\Pi-22 \%}=54,3 ; \square_{\Pi-30 \%}=44,55 ; \square_{\Pi-38 \%}=34,8 .
$$

Как известно с ростом температуры коэффициент теплопроводности так же уменьшается. Расчетные данные по формуле 7 объясняют наличие зоны плавления на образцах с пористостью $\Pi=38 \%$ при плотности мощности не ниже $0,8 \cdot 10^{4} \quad \mathrm{BT} / \mathrm{cm}^{2}$ и отсутствие зоны оплавления на образцах с пористостью П=22\% в интервале плотности мощности $0,8-1,2 \cdot 10^{4} \mathrm{BT} / \mathrm{cm}^{2}$ и скорости обработки выше $10 \mathrm{mм} / \mathrm{c}$. Снижение коэффициента теплопроводности с увеличением объемной доли пор способствует изоляции теплоты переданной поверхности лазерным излучением и приводит к плавлению материала.

Тройное взаимодействие факторов представляют собой некий структурноэнергетический параметр лазерной обработки пористых материалов (уравнение 1). Взаимодействие плотности потока энергии лазерного излучения и пористости увеличивает ширину оплавленной зоны, очевидно, в основном за счет влияния пористости на теплопроводность
Теплопроводность железа составляет 81 Вт/м·К (при 293,15 К) [2, 3] и расчет по формуле 7 дает численные значения теплопроводности в зависимости от пористости железных прессовок участвующих в матрице планирования (таблица 2):

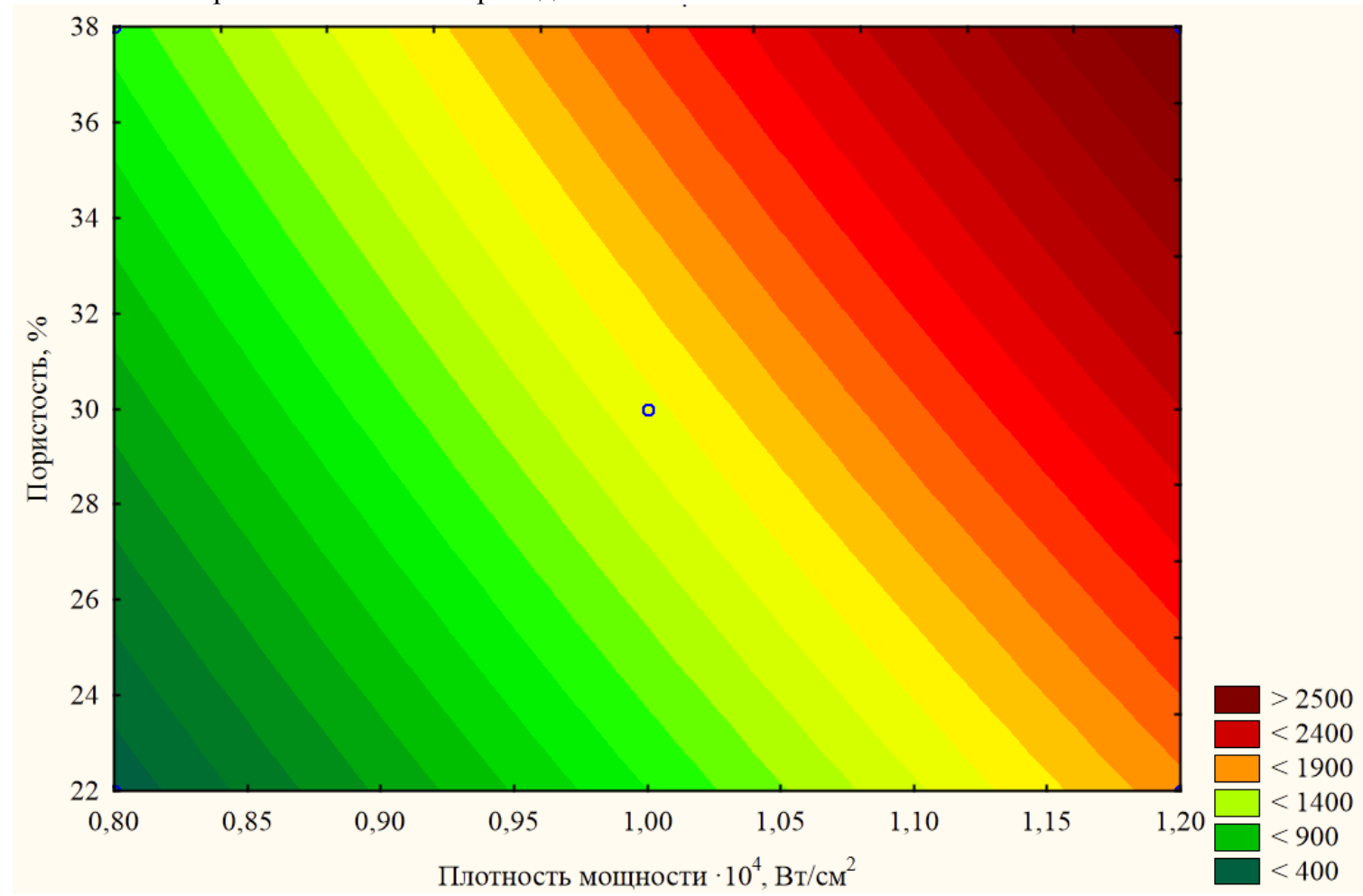

Рисунок 8 - Проекции изолиний ширины оплавленной зоны на плоскость $\left(x_{4}=0,73\right.$, скорость обработки 15 мм/с)

материала. На рисунке 8 представлены проекции изолиний ширины оплавленной зоны в координатах плотность мощности - пористость.

Рассматривая изменение глубины оплавленной зоны (уравнение 2), необходимо заметить, что коэффициент парного взаимодействия плотности мощности и скорости обработки оказывается статистически не значимым. На распространение фронта плавления вглубь материала от действия лазерного излучения плазменные процессы, развивающиеся с увеличением плотности потока энергии, протекающие на поверхности, не оказывают заметного влияния.

В отдельности коэффициенты регрессионного уравнения 2, отражающие влияние плотности мощности лазерного излучения и скорости обработки, оказывают аналогичное качественное влияние, что и на ширину зоны плавления (уравнение 1). 
Определяющее значение на глубину зоны плавления (уравнение 2) оказывает пористость, с увеличением которой глубина зоны плавления увеличивается, очевидно, по причинам рассмотренным выше. Проекции изолиний ширины оплавленной зоны в координатах плотность мощности - пористость представлены на рисунке 9.

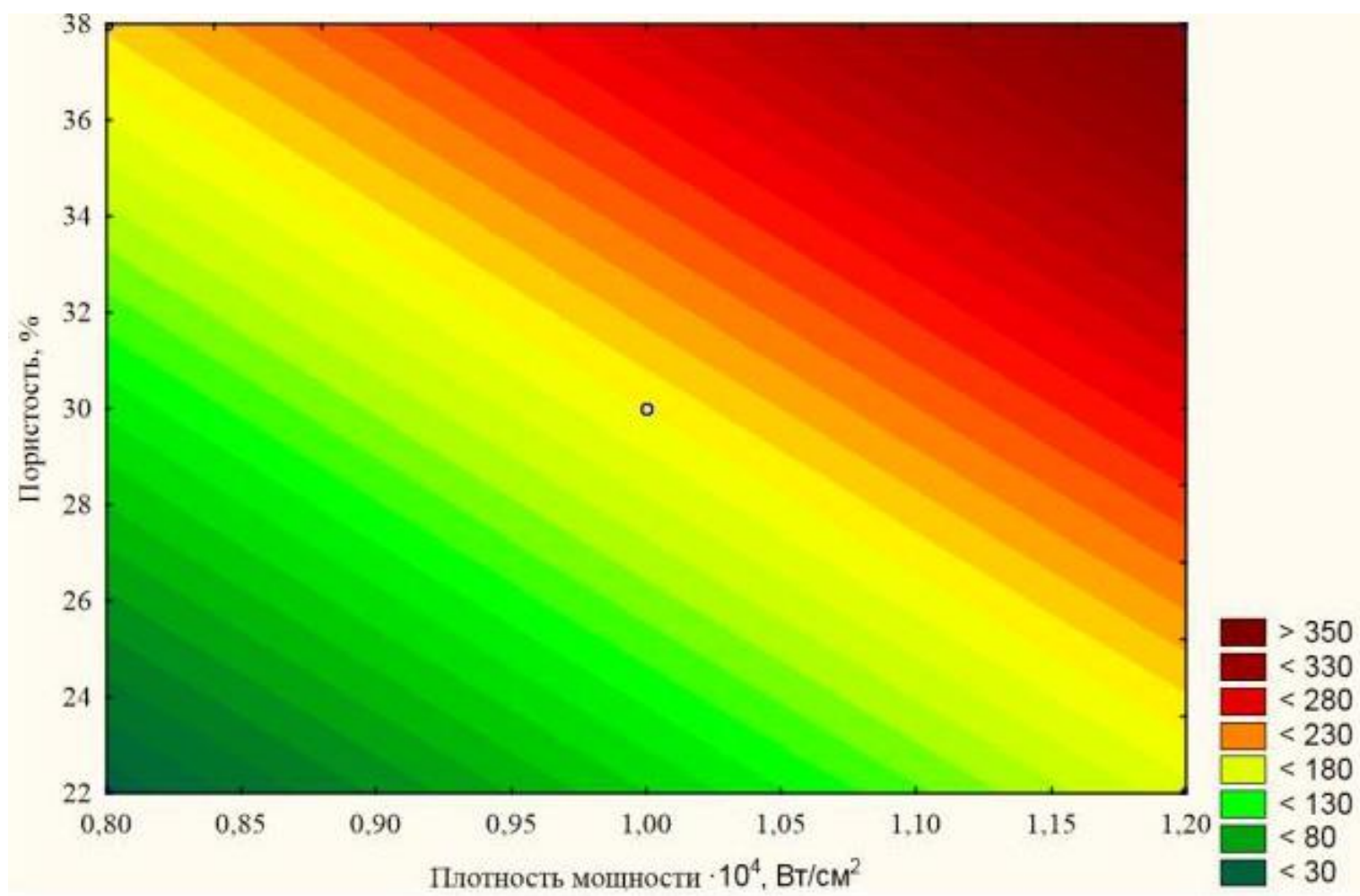

Рисунок 9 - Проекции изолиний глубины оплавленной зоны на плоскость $\left(x_{u}=0,73\right.$, скорость обработки 15 мм/с)

Зона термического влияния образуется под действием лазерного излучения при всех энергетических и структурных параметрах в проведенных опытах, в том числе и в отсутствие зоны плавления. Большие размеры ЗТВ по сравнению с зоной плавления и наличие ЗТВ во всех проведенных опытах, очевидно, связано со значительно меньшим энерговкладом, требующимся для ее образования.

Наиболее ощутимое влияние на ширину ЗТВ оказывает коэффициент парного взаимодействия плотности мощности и скорости обработки уменьшающий ширину ЗТВ, что, как указано выше, связано с плазменными процессами на поверхности при лазерной обработке.

Коэффициенты уравнения регрессии 3, отражающие отдельное влияние плотности мощности, скорости обработки и пористости, проявляют аналогичное с зоной плавления качественное воздействие на ширину ЗТВ.

Тройное взаимодействие плотности мощности, скорости обработки и пористости уменьшают ширину ЗТВ (уравнение 3). Пористость препятствует распространению теплоты в материале, проявляя изолирующее для теплоты точечного источника действие в твердой фазе.

Небольшое влияние на рост ширины ЗТВ имеет парное взаимодействие скорости обработки и пористости, также представляющее собой структурно-энергетический фактор процесса формирования ЗТВ в условиях действия точечного источника теплоты. Также, как и указано выше, определяющее значение в рассматриваемом парном взаимодействии, очевидно, проявляет пористость. На рисунке 10 представлено изменение ширины ЗТВ в координатах плотность мощности - скорость обработки.

На глубину ЗТВ так же влияют все структурно-энергетические характеристики в рассматриваемой матрице планирования экспериментов (уравнение 4). Наибольшее влияние на глубину ЗТВ, как и на глубину зоны оплавления, оказывает пористость, увеличивая глубину ЗТВ. Парное взаимодействие плотности мощности и скорости обработки уменьшает глубину 3ТВ. При этом влияние пористости по модулю немного больше, чем парное взаимодействие плотности мощности и скорости обработки. Очевидно, что плазменные процессы, 


\begin{tabular}{l|lr|ll|ll} 
& ISRA (India) & $=\mathbf{1 . 3 4 4}$ & SIS (USA) & $=\mathbf{0 . 9 1 2}$ & ICV (Poland) & $=\mathbf{6 . 6 3 0}$ \\
Impact Factor: & ISI (Dubai, UAE) $=\mathbf{0 . 8 2 9}$ & PUH (Russia) & $=\mathbf{0 . 2 3 4}$ & PIF (India) & $=\mathbf{1 . 9 4 0}$ \\
& GIF (Australia) & $=\mathbf{0 . 5 6 4}$ & ESJI (KZ) & $=\mathbf{1 . 0 4 2}$ & IBI (India) & $=\mathbf{4 . 2 6 0}$
\end{tabular}

развивающиеся на поверхности, оказывают достаточно сильное влияние на глубину ЗТВ в отличие от глубины оплавленной зоны, где этот фактор оказывается статистически незначимым.
На рисунке 11 представлено изменение глубины ЗТВ в координатах плотность мощности пористость.

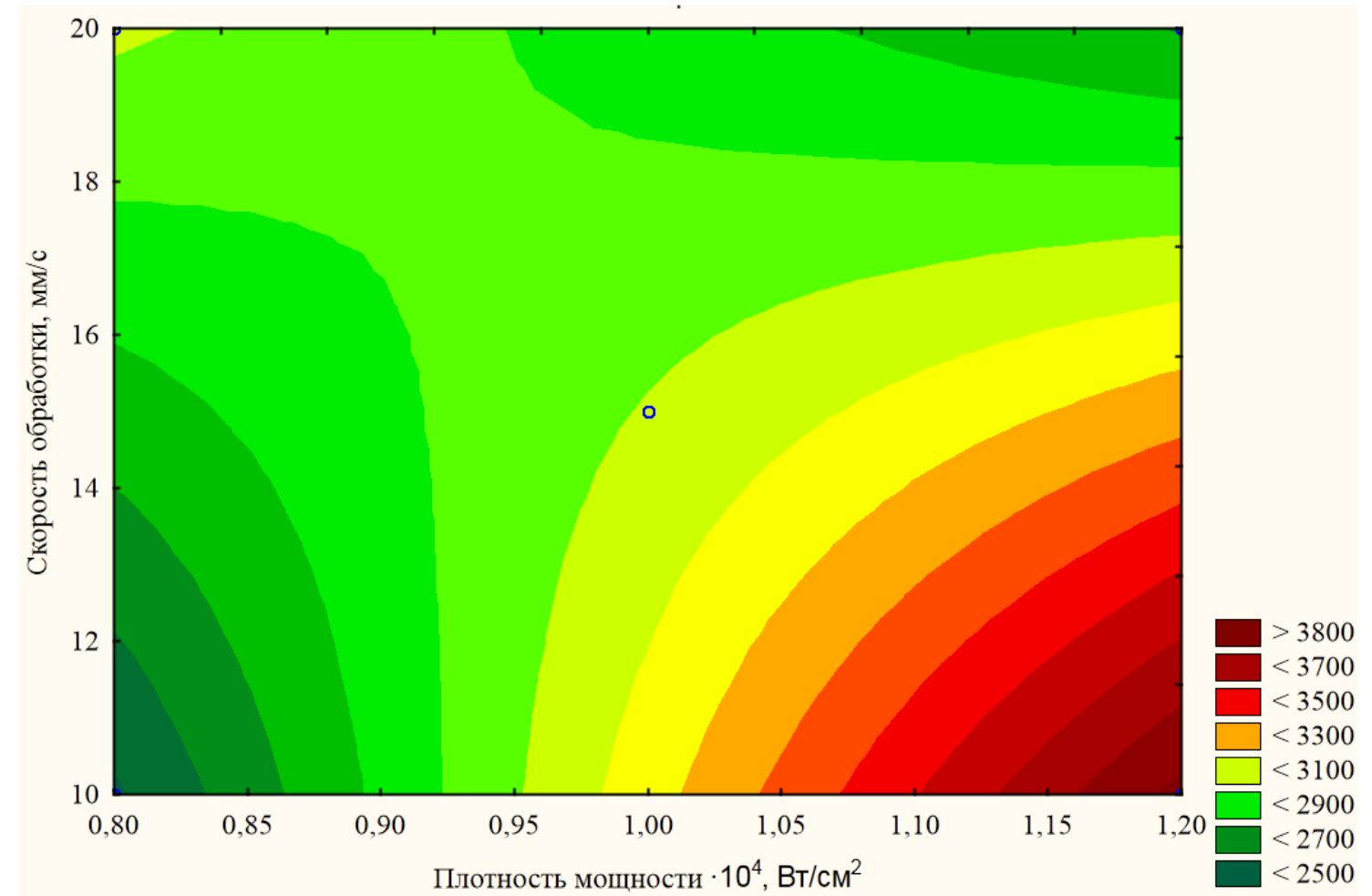

Рисунок 10 - Проекции изолиний ширины ЗТВ на плоскость $\left(x_{4}=0,73, \Pi=30 \%\right)$

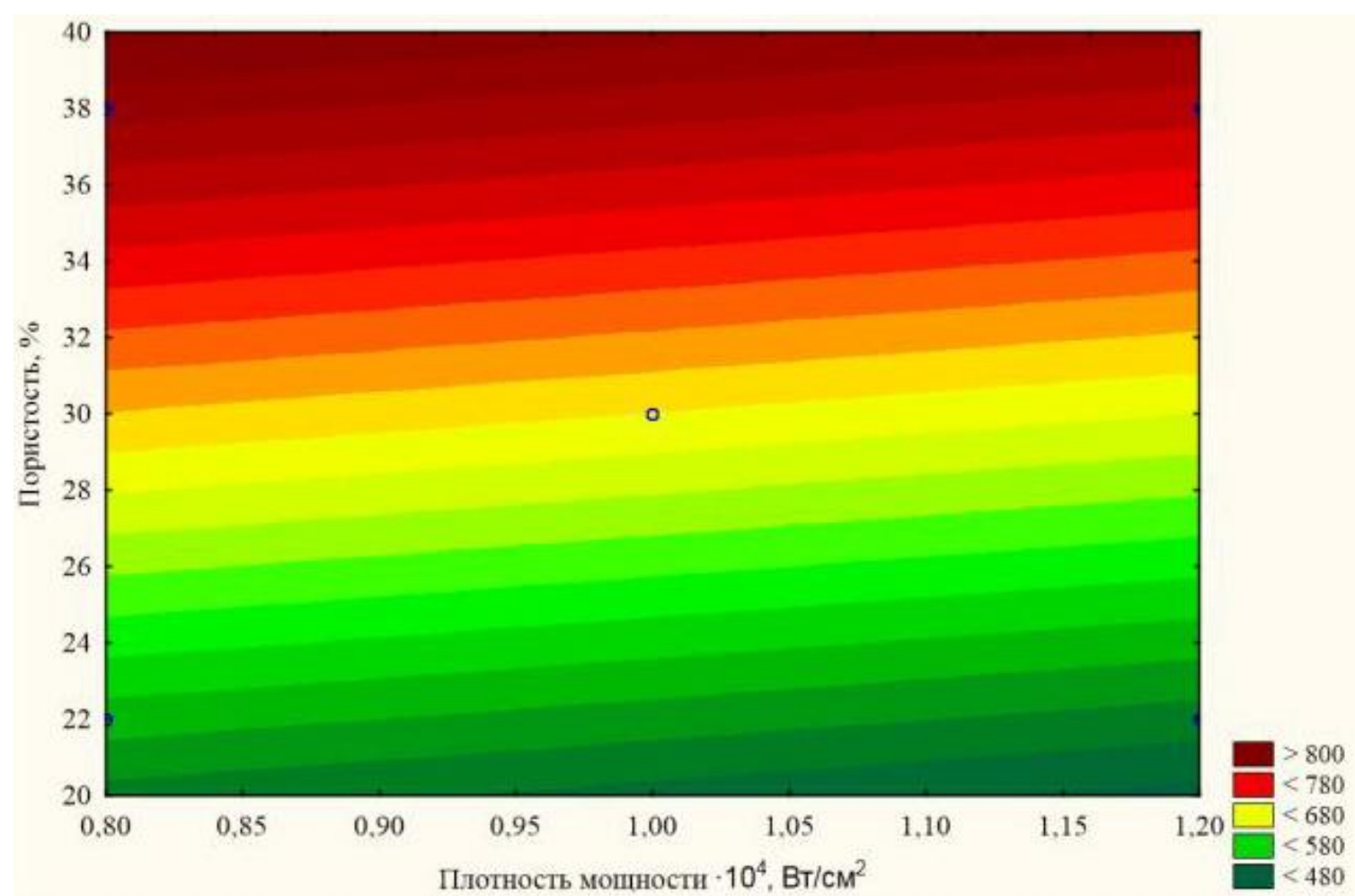

Рисунок 11 - Проекции изолиний ширины ЗТВ на плоскость $\left(x_{u}=0,73\right.$, скорость обработки 15 мм/с)

ISPC Technology and science,

Philadelphia, USA 


\begin{tabular}{|c|c|c|c|c|c|c|}
\hline Impact Factor: & $\begin{array}{l}\text { ISRA (India) } \\
\text { ISI (Dubai, UAF } \\
\text { GIF (Australia) } \\
\text { JIF }\end{array}$ & $\begin{array}{l}=1.344 \\
=0.829 \\
=0.564 \\
=1.500\end{array}$ & $\begin{array}{l}\text { SIS (USA) } \\
\text { PИНЦ (Russia) } \\
\text { ESJI (KZ) } \\
\text { SJIF (Morocco) }\end{array}$ & $\begin{array}{l}=0.912 \\
=0.234 \\
=1.042 \\
=2.031\end{array}$ & $\begin{array}{l}\text { ICV (Poland) } \\
\text { PIF (India) } \\
\text { IBI (India) }\end{array}$ & $\begin{array}{l}=6.630 \\
=1.940 \\
=4.260\end{array}$ \\
\hline
\end{tabular}

\section{Выводы:}

1. Детально рассмотрено влияние энергетических и структурных факторов на формирование зоны оплавления и ЗТВ при обработке лазерным излучением пористых спеченных материалов из восстановленного карбонильного железа.

2. Установлено, что структурноэнергетические параметры обработки нелинейно воздействуют на размеры оплавленной зоны и ширину ЗТВ.

3. Отдельное влияние плотности мощности и скорости обработки хорошо согласуются с ранее проведенными исследованиями для компактных материалов. Плотность мощности прямопропорционально увеличивает, а скорость обработки уменьшает размеры зоны оплавления и ЗТВ.

4. Пористость увеличивает размеры зоны плавления и ЗТВ, что связано с ее влиянием на теплофизические характеристики материалов, в частности, пористость снижает коэффициент теплопроводности и способствует концентрации теплоты в небольшом объеме от точечного источника.
5. Парное взаимодействие плотности мощности и скорости обработки, представляющее собой плотность потока энергии, уменьшает размеры зоны плавления и 3ТВ, особенно сильное влияние это парное взаимодействие оказывает на ширину ЗТВ. Такое воздействие плотности потока энергии связывается с плазменными процессами, возникающими на поверхности обрабатываемого материала под действием лазерного излучения.

6. Парное взаимодействие скорости обработки и пористости является структурноэнергетическим параметром в условиях воздействия лазерного излучения, оказывается статистически значимым лишь для ширины 3ТВ, имеет небольшое воздействие, увеличивая ее.

7. Тройное взаимодействие плотности мощности, скорости обработки и пористости увеличивает размеры зоны оплавления и уменьшает ширину ЗТВ. На указанные параметры отклика системы тройное взаимодействие имеет небольшое влияние. Изменение качественного характера воздействия на параметры отклика, очевидно, связано с влиянием пористости с присутствием или отсутствием жидкой фазы в системе.

\section{References:}

1. Libensov GA, Lopatin VJ, Komarnickij GV (2002) Processy poroshkovoj metallurgii. V 2-h t. T.2. Formovanie i spekanie: Uchebnik dlja vuzov. - M.: MISIS, 2002 - 320 p.

2. (1987) Poristye pronicaemye materialy: Sprav.izd. / Pod red. Belova S.V. M.: Metallurgija, 1987. 335 p.

3. Sorokin VK, Kostromin SV, Beljaev ES (2016) Tehnologii i svojstva poroshkovyh materialov. - Saarbrücken: LAP LAMBERT Academic Publishing, 2016. - 76 p.

4. Kikin PJ (2009) Vremennoj sdvig tochek plavlenija $\mathrm{i}$ isparenija $\mathrm{v}$ ul'tramelkozernistom aljuminievom splave pri lazernom nagreve [Tekst] / Kikin P.Ju., Pchelincev A.I., Rusin E.E. // Fizika i himija obrabotki materialov. M.2009. - №2. p.50-53.

5. Maranc AV (2013) Lazernaja obrabotka spechennoj poroshkovoj stali SPN14A7M5 / Maranc A. V., Sentjurina Zh. A., Jadrojceva I. A., Narva V. K., Smurov I. Ju. [Tekst] // Izvestija vysshih uchebnyh zavedenij.
Poroshkovaja metallurgija i funkcional'nye pokrytija. M.-2013. - №2. p.19-25.

6. Beljaev ES (2010) Issledovanie struktury i svojstv lazernyh svarnyh soedinenij poristogo pronicaemogo prokata iz karbonil'nogo nikelevogo poroshka [Tekst] / Beljaev E.S., Gavrilov G.N. // «Tehnologija metallov». M.2010. - №12. p.28-33.

7. Beljaev ES (2010) Issledovanija struktury i svojstv lazernyh svarnyh soedinenij korrozionno-stojkoj stali 12H18N10T i poristogo pronicaemogo prokata iz karbonil'nogo poroshka nikelja [Tekst] / Beljaev E.S., Gavrilov G.N., Hrenov V.A.// «Tehnologija metallov». M.-2010. - № 10. p. 30-34.

8. Beljaev ES (2010) Metallovedcheskie osnovy poluchenija soedinenij korrozionno - stojkoj stali i poristyh nikelevyh materialov lazernym izlucheniem pri proizvodstve fil'trov: dis. ... kand. tehn. nauk. Nizhegorod. gos. tehn. universitet im. R.E. Alekseeva, Nizhnij Novgorod, 2010. 


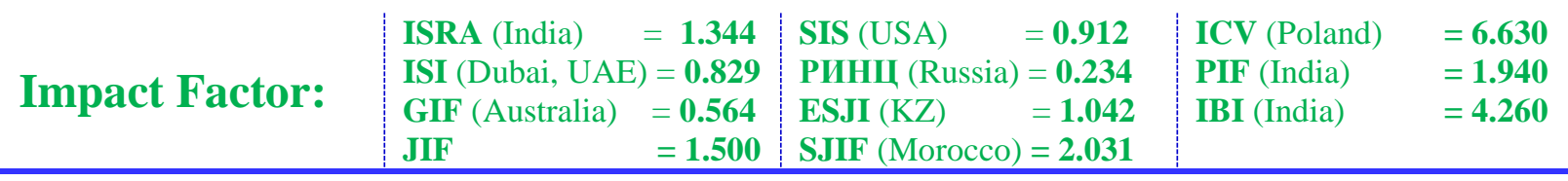

9. Beljaev ES (2013) Mikrostruktura i svojstva svarnyh soedinenij iz poristogo prokata poluchennye izlucheniem lazera [Tekst] / Beljaev E.S., Kostromin S.V. // «Aktual'nye problemy gumanitarnyh i estestvennyh nauk». M.-2013. - №2. p.34-37.

10. Grigor'janc AG, Shiganov IN, Misjurov AI (2006) Tehnologicheskie processy lazernoj obrabotki: Ucheb. Posobie dlja vuzov / Pod red.
A.G.Grigor'janca.-M.: Izd-vo MGTU im. N.Je.Baumana, 2006. -664 p.

11. Beljaev ES (2016) Primenenie metoda matematicheskogo planirovanija jeksperimenta pri izuchenii processa kompleksnoj termicheskoj obrabotki stali 38HN3MFA [Tekst]/ Beljaev E.S., Tumina E.V., Makarov N.V// Theoretical \& Applied Science. 2016. № 11 (43). p. 118-126 